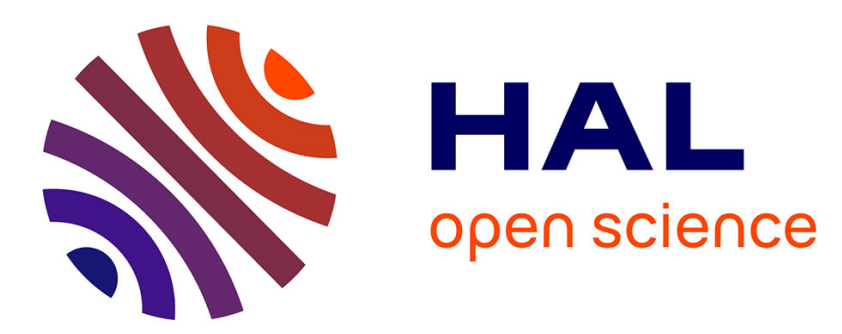

\title{
Sinusite invasive à Scedosporium Apiospermum révélée par un abcès extradural
}

\author{
N. Khoueir, B. Verillaud, P. Herman
}

\section{To cite this version:}

N. Khoueir, B. Verillaud, P. Herman. Sinusite invasive à Scedosporium Apiospermum révélée par un abcès extradural. Annales françaises d'Oto-rhino-laryngologie et de Pathologie Cervico-faciale, 2019, 136, pp.121 - 123. 10.1016/j.aforl.2018.06.004 . hal-03486572

\section{HAL Id: hal-03486572 \\ https://hal.science/hal-03486572}

Submitted on 20 Dec 2021

HAL is a multi-disciplinary open access archive for the deposit and dissemination of scientific research documents, whether they are published or not. The documents may come from teaching and research institutions in France or abroad, or from public or private research centers.
L'archive ouverte pluridisciplinaire HAL, est destinée au dépôt et à la diffusion de documents scientifiques de niveau recherche, publiés ou non, émanant des établissements d'enseignement et de recherche français ou étrangers, des laboratoires publics ou privés.

\section{다)(1) $(5$}

Distributed under a Creative Commons Attribution - NonCommerciall 4.0 International 
Sinusite invasive à Scedosporium Apiospermum révélée par un abcès extradural.

Nadim Khoueir, MD (1) (2) ; Benjamin Verillaud MD, PhD (1); Philippe Herman, MD, PhD (1)

(1)Département d'Otorhinolaryngologie/Chirurgie cervico-faciale/Chirurgie de la base du crâne Hôpital Lariboisière, APHP

Université Paris-Diderot,

Paris, France.

(2)Département d'Otorhinolaryngologie/Chirurgie cervico-faciale

Hôpital Universitaire Hôtel Dieu de France

Université Saint Joseph, Faculté de Médecine

Beyrouth, Liban

Auteur correspondant : Nadim Khoueir,

Adresse: 2 Rue Ambroise Paré, 75010, Paris, France

Tel: +33617493562

Courriel : nadim_khoueir@hotmail.com

Conflit d'intérêts : Aucun

Financement : Aucun 


\section{RESUME}

Introduction : La rhinosinusite fongique invasive chronique (RSFIC) est une entité rare qui affecte généralement les sujets immunodéprimés. L'agent pathogène le plus fréquemment retrouvé est Aspergillus. Elle prend le plus souvent à l'imagerie la forme d'une pathologie agressive pseudo-tumorale. Nous présentons un cas de RSFIC à Scedosporium Apiospermum (S.Apiospermum) avec une présentation clinique et radiologique atypique.

Résumé du cas : Un homme immunocompétent âgé de 72 ans présentait des céphalées chroniques, des cervicalgies et une limitation bilatérale dans le regard latéral. L'imagerie révélait une pathologie sphénoïdale gauche isolée avec des remaniements osseux importants et un abcès extradural en regard du clivus. Une sphénoïdotomie endoscopique large avec rhinopharyngectomie de type II était réalisée et le diagnostic de RSFIC à S.Apiospermum était posé sur les données de l'histologie et de la culture fongique. Le patient refusait tout traitement médical. Il n’y avait pas de récidive après 1 an de suivi.

Discussion : S.Apiospermum est un germe rarement isolé dans la RSIFC. Notre cas était révélé par une présentation clinique atypique incluant une infection sphénoïdale isolée compliquée par une paralysie bilatérale du VI et un abcès extradural. L'imagerie était également inhabituelle, prenant plutôt l'aspect d'une dysplasie fibreuse ou d'une ostéomyélite bactérienne que l'aspect pseudo-tumoral typique. Le patient était traité avec succès par chirurgie seule, qui pourrait donc être suffisante chez les sujets immunocompétents.

Mots clés : rhinosinusite fongique invasive; abcès extradural; sinus sphénoïdal. 


\section{INTRODUCTION}

On distingue classiquement parmi les rhinosinusites fongiques les formes non invasives et invasives. Trois formes de rhinosinusite fongique invasive ont été décrites par DeShazo et al : les formes aiguë fulminantes, granulomateuses et chroniques ${ }^{1}$. La rhinosinusite fongique invasive chronique (RSFIC) est définie par une invasion fongique de la sous-muqueuse associée à une inflammation chronique et à une fibrose, évoluant pendant plus de 12 semaines. Les patients affectés sont généralement immunocompétents, mais ceux qui présentent une immunosuppression relative comme le diabète peuvent également être touchés ${ }^{2}$. Les agents pathogènes les plus fréquemment en cause sont les espèces d'Aspergillus, les moisissures

dématiacées et les mucomycoses ${ }^{3}$. Scedosporium Apiospermum (S.Apiospermum) est un agent pathogène rare retrouvé dans les eaux polluées, les eaux usées et les sols ${ }^{4}$. Bien qu'il provoque rarement des infections significatives chez les patients immunocompétents, il peut être associé à des infections invasives graves chez les hôtes immunodéprimés ${ }^{4}$.

Le cas présenté ici est celui d'une présentation clinique atypique de RSFIC à S. Apiospermum chez un patient immunocompétent.

L'objectif de ce rapport est de présenter le cas et de discuter les diagnostics différentiels et le traitement.

\section{RESUME DU CAS}

Un homme immunocompétent âgé de 72 ans était adressé dans le service pour des céphalées croissantes depuis 4 mois associées à des cervicalgies bilatérales et à une limitation progressive du regard latéral des 2 côtés. Il avait reçu plusieurs cures d'antibiotiques, sans amélioration. Sur le plan des symptômes nasosinusiens, il décrivait une congestion et une rhinorrhée postérieure. A 
l'examen, il existait une paralysie bilatérale dans le regard latéral avec des réflexes pupillaires normaux. L'endoscopie nasale montrait une légère congestion muqueuse. Sur le scanner, le sphénoïde était asymétrique (Figure 1). A droite, le sinus était normalement pneumatisé. A gauche, l'os était épaissi au niveau du corps sphénoïdal, de la base des ptérygoïdes et de la grande aile du sphénoïde en dehors du foramen rond. Une lyse osseuse irrégulière était notée sur le clivus, le condyle occipital, le second genou de l'artère carotide interne gauche et le plancher sellaire. L'IRM montrait que cette lyse osseuse communiquait avec une collection extradurale isointense en $\mathrm{T} 1$ et $\mathrm{T} 2$ et se rehaussant en périphérie après injection de gadolinium (Figure 2). Une exploration chirurgicale était réalisée sous anesthésie générale et sous neuronavigation. Dans un premier temps, une sphénoïdotomie trans-ethmoïdale droite était effectuée avec une septectomie postérieure : l'objectif était de trouver des repères anatomiques chez ce patient présentant une anatomie remaniée au niveau du sinus sphénoïdal gauche. La cavité sphénoïdale gauche, siège d'une rétention purulente, était identifiée après un fraisage prolongé. Une rhinophayngectomie de type II était ensuite réalisée afin de drainer l'abcès clival en contact intime avec la dure-mère (Figure 3). La résection incluait la paroi nasopharyngée postérosupérieure jusqu'au fascia pharyngobasilaire, le rostre sphénoïdal et le septum intersphénoïdal, ainsi que la paroi antérieure et le plancher du sinus sphénoïdal. La dure-mère était finalement recouverte par un lambeau nasoseptal droit. L'examen histologique des prélèvements retrouvait des signes d'inflammation muqueuse chronique non spécifique avec une fibrose et un léger remodelage osseux. Des filaments fongiques étaient mis en évidence dans l'espace sousmuqueux. La culture était positive pour S.Apiospermum, sensible à de nombreux antifongiques dont l'amphotéricine B et le voriconazole. La culture bactériologique était négative, chez un patient n'ayant reçu aucun antibiotique en préopératoire. Le diagnostic retenu était celui d'une 
RSFIC compliquée d'abcès extradural. Un traitement par voriconazole per-os était proposé, mais refusé par le patient. A un an de suivi, le patient était asymptomatique et il n'y avait pas de signe de récidive ni à l'examen clinique ni à l'IRM.

\section{DISCUSSION}

Le diagnostic de RSFIC était posé ici devant la durée prolongée des symptômes, les résultats histologiques et la culture fongique. Les sinusites à S.Apiospermum sont rares. Dans leur série de 400 sinusites fongiques, Montone et al. ont isolé S.Apiospermum dans 5\% des aspergillomes et dans un seul cas de RSFIC ${ }^{2}$.

Outre la rareté de la RSFIC et du pathogène isolé, notre cas était remarquable par sa présentation inhabituelle : pathologie sphénoïdale gauche isolée avec remodelage osseux important, abcès extradural en regard du clivus et paralysie bilatérale du 6ème nerf crânien chez un patient immunocompétent. Les diagnostics différentiels possibles incluaient le chondrosarcome, le chordome, la dysplasie fibreuse, le carcinome et l'infection ${ }^{5}$. La dysplasie fibreuse affecte les patients plus jeunes et implique rarement le sinus sphénoïdal de façon isolée. Cependant, il existait au scanner dans notre cas un aspect en « verre dépoli » de l'os, avec un kyste anévrysmal intra-osseux associé. L'érosion du clivus pouvait orienter vers un chordome. Cependant le chordome ne présente habituellement pas cet aspect d'épaississement osseux avec un contour préservé, et le rehaussement après gadolinium est plutôt hétérogène avec un aspect en «nid d'abeilles $»^{7}$. L'abcès extradural est une complication classique des sinusites frontales : elle est

exceptionnelle dans les sinusites ethmoïdales et sphénoïdales ${ }^{5 ; 8 ; 9}$, et concerne alors des sinusites d'origine bactérienne, sans qu'il soit fait mention d'une atteinte d'un nerf crânien. L'aspect radiologique de la RSFIC est souvent aspécifique. Elle peut consister en un simple 
épaississement muqueux, même si la présentation la plus fréquente est celle d'un comblement tissulaire dans un ou plusieurs sinus paranasaux avec une érosion osseuse et une invasion des espaces environnants ${ }^{10}$. Cet aspect peut aussi orienter vers une tumeur maligne et il est souvent difficile de différencier ces entités uniquement sur les données de l'imagerie. Le cas présenté ici était atypique dans la mesure où il n'existait pas à l'imagerie d'aspect «pseudo-tumoral», agressif. Cela pourrait être lié au fait que le pathogène isolé, S.Apiospermum, puisse avoir un comportement invasif différent de celui des pathogènes plus fréquents que sont Aspergillus, les moisissures dématiacées ou les mucomycoses ${ }^{3}$.

Il n'existe pas de consensus bien établi concernant la prise en charge optimale de la RSFIC, mais la plupart des auteurs proposent une combinaison de débridement chirurgical, de traitement antifongique systémique adapté à l'antifongigramme, et le cas échéant de correction maximale de l'immunosuppression ${ }^{3}$. Dans notre cas, le patient refusait le traitement par voriconazole, et avec un recul d'un an, la chirurgie seule semblait avoir permis de contrôler la maladie chez ce patient immunocompétent.

\section{CONCLUSION}

En conclusion, nous présentons un cas rare de RSFIC secondaire à S.Apiospermum chez un patient immunocompétent avec une présentation clinique et radiologique atypique. La chirurgie permet les prélèvements indispensables au diagnostic, et pourrait constituer une option thérapeutique suffisante chez les patients immunocompétents.

Conflit d'intérêts: Pas de conflit d'intérêt

\section{REFERENCES:}


1-DeShazo RD, O’Brien M, Chapin K, et al. A new classification and diagnostic criteria for invasive fungal sinusitis. Arch Otolaryngol Head Neck Surg. 1997 Nov;123(11):1181-8

2-Montone KT, Livolsi VA, Feldman MD et al. Fungal rhinosinusitis: a retrospective microbiologic and pathologic review of 400 patients at a single university medical center. Int J Otolaryngol. 2012;2012:684835.

3- Thompson GR 3rd, Patterson TF. Fungal disease of the nose and paranasal sinuses. J Allergy Clin Immunol. 2012 Feb;129(2):321-6.

4- Baumgartner BJ, Rakita RM, Backous DD. Scedosporium apiospermum otomycosis. Am J Otolaryngol. 2007 Jul-Aug;28(4):254-6.

5-Hoistad DL, Duvall AJ 3rd. Sinusitis with contiguous abscess involvement of the clivus and petrous apices. Case report. Ann Otol Rhinol Laryngol. 1999 May;108(5):463-6.

6-Ferguson BJ. Fibrous dysplasia of the paranasal sinuses. Am J Otolaryngol. 1994 MayJun;15(3):227-30.

7-Erdem E, Angtuaco EC, Van Hemert R, Park JS, Al Mefty O. Comprehensive review of intracranial chordoma. Radiographics. 2003 Jul-Aug;23(4):995-1009

8- Karatas A, Is M, Guclu E, Dosoglu M, Gezen F. Intracranial epidural abscess secondary to isolated sphenoid sinusitis. Br J Neurosurg. 2007 Dec;21(6):616-8.

9- Moonis G, Granados A, Simon SL. Epidural hematoma as a complication of sphenoid sinusitis and epidural abscess a case report and literature review. Clin Imaging. 2002 Nov-Dec;26(6):3825.

10-Aribandi M, McCoy VA, Bazan C. Imaging features of invasive and noninvasive fungal sinusitis: a review. Radiographics. 2007 Sep-Oct;27(5):1283-96 
Figure 1: Tomodensitométrie en coupe coronale montrant une sclérose osseuse de l'os sphénoïde à gauche.

Figure2 : IRM en coupe sagittale en séquence pondérée $\mathrm{T} 1$ avec injection de gadolinium montrant une collection extradurale en regard du clivus avec prise de contraste périphérique.

Figure 3: Drainage de l'abcès en regard du clivus avec exposition de la dure-mère (étoile noire). Noter l'aspect du sinus sphénoïdal gauche après fraisage (étoile blanche), et le reliquat de rostre sphénoïdal (flèche noire). 


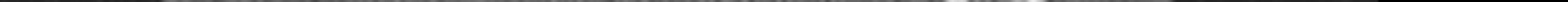




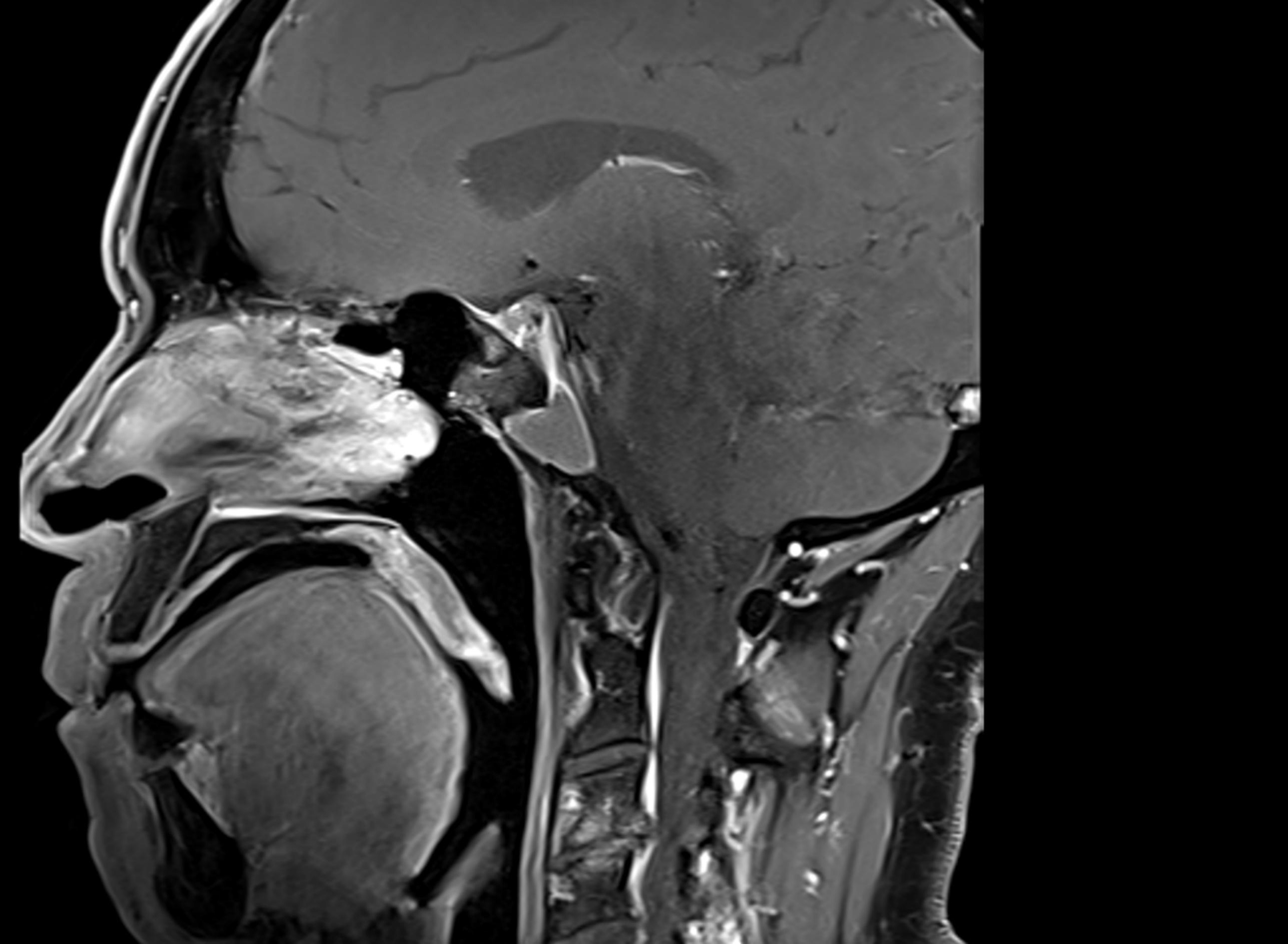




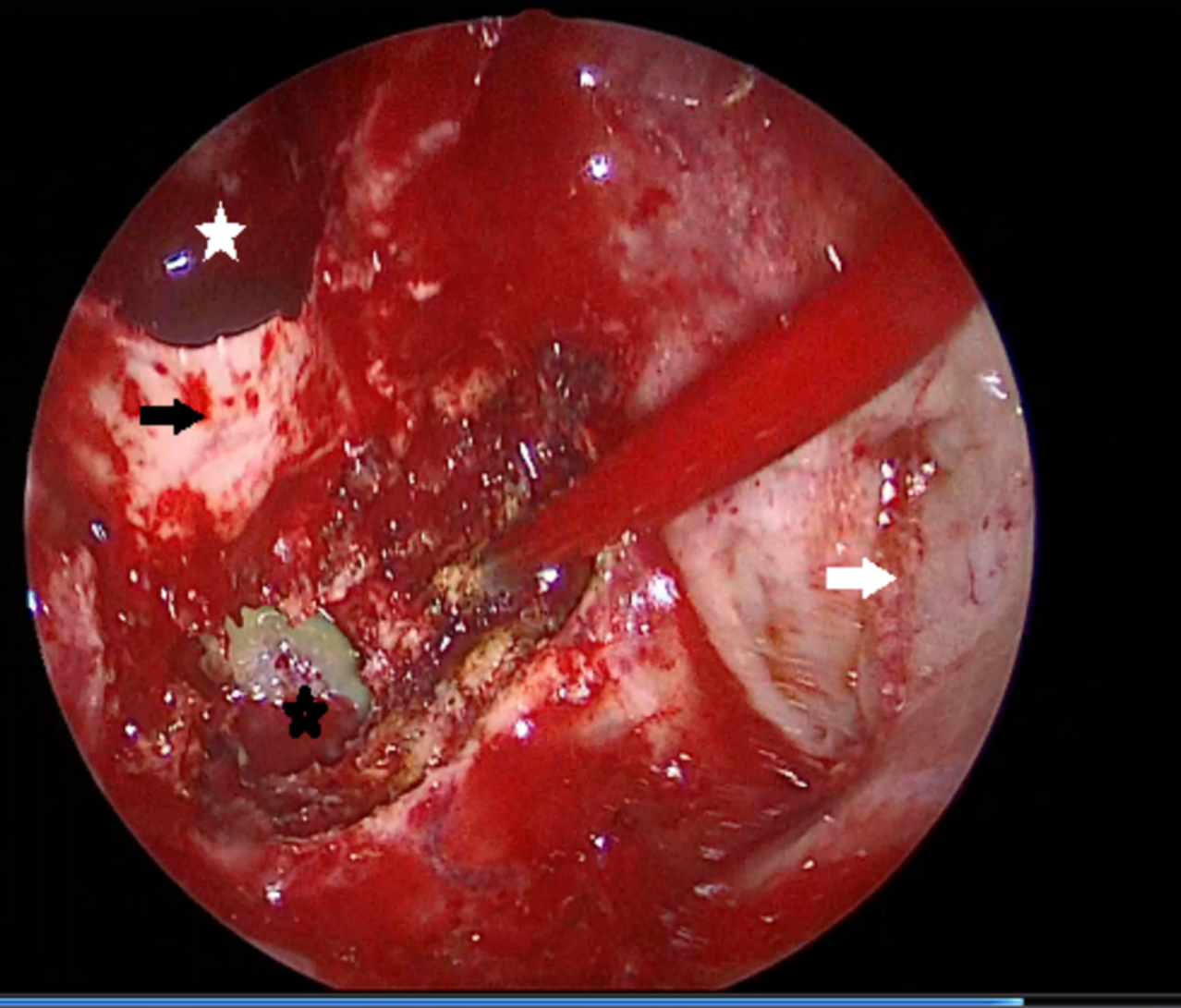

